\title{
Sex-specific associations of insulin resistance with chronic kidney disease and kidney function: a bi-directional Mendelian randomisation study
}

\author{
Jie V. Zhao ${ }^{1}$ (D) C. Mary Schooling ${ }^{1,2}$ (D) \\ Received: 14 January 2020 / Accepted: 24 March 2020 / Published online: 15 May 2020 \\ (C) Springer-Verlag GmbH Germany, part of Springer Nature 2020
}

\begin{abstract}
Aims/hypothesis Reasons for the sexual disparity in chronic kidney disease (CKD) are unclear. To provide insight we contextualised these differences within evolutionary biology, and explored sex-specific effects of insulin resistance because it may have sex-specific effects on the reproductive axis. Impaired kidney function may also cause insulin resistance. We assessed these possibilities using bi-directional, sex-specific, two-sample Mendelian randomisation (MR).

Methods Given that fasting insulin, fasting glucose and $\mathrm{HbA}_{1 \mathrm{c}}$ are related, we used MR-Bayesian model averaging (MR-BMA) to identify the best-fitting model and most influential exposure. Genetic associations with glycaemic traits were obtained from genome-wide association studies (GWAS) in Europeans without diabetes ( $n=108,557$ for fasting insulin, as a proxy for insulin resistance, and for fasting glucose, $n=123,665$ for $\mathrm{HbA}_{1 \mathrm{c}}$ in the Meta-Analyses of Glucose and Insulin-related traits Consortium [MAGIC]), and applied to GWAS of 480,698 Europeans for overall associations with CKD (cases $n=41,395)$ and eGFR. We also used sex-specific individual information in white British (179,917 men, 6016 CKD cases; 212,079 women, 5958 CKD cases) from the UK Biobank. Univariable or multivariable MR was used to assess the role of glycaemic trait(s) selected by MRBMA in CKD and kidney function. Genetic variants predicting eGFR were used to assess the role of kidney function in the most influential exposure(s).

Results Fasting insulin was selected as the most likely exposure by both overall and sex-specific MR-BMA. It increased CKD in men (OR 7.23 per pmol/1 higher fasting insulin [95\% CI 2.46, 21.2]) but not in women (OR 1.05 [95\% CI 0.21, 5.21]), and reduced eGFR in men $(-0.04[95 \% \mathrm{CI}-0.07,-0.01])$ but not in women $(0.01[95 \% \mathrm{CI}-0.02,0.03])$. Genetically predicted eGFR was unrelated to fasting insulin.

Conclusions/interpretation Genetically predicted fasting insulin was sex-specifically associated with CKD and unhealthier kidney function but was not affected by kidney function.
\end{abstract}

Keywords Insulin resistance $\cdot$ Mendelian randomisation $\cdot$ Renal function $\cdot$ Sex disparity

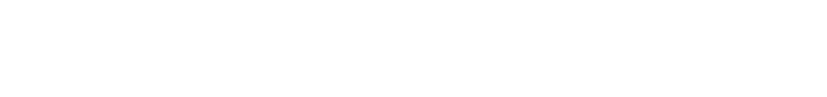

Electronic supplementary material The online version of this article (https://doi.org/10.1007/s00125-020-05163-y) contains supplementary material, which is available to authorized users.

Jie V. Zhao

janezhao@hku.hk

1 School of Public Health, Li Ka Shing Faculty of Medicine, The University of Hong Kong, 1/F, Patrick Manson Building, 7 Sassoon Road, Hong Kong, SAR, China

2 City University of New York, School of Public Health and Health Policy, New York, NY, USA

\section{Abbreviations}

CKD

CKDGen

GWAS

IVW

MAGIC

MR

MR-BMA

MR-PRESSO
Chronic kidney disease

Chronic Kidney Disease Genetics Genome-wide association study(ies) Inverse variance weighting Meta-Analyses of Glucose and Insulin-related traits Consortium Mendelian randomisation MR-Bayesian model averaging Mendelian Randomisation Pleiotropy Residual Sum and Outlier 


\section{Research in context}

\section{What is already known about this subject?}

- There is a sex disparity in chronic kidney disease (CKD), with men being more severely affected than women

- Insulin resistance might be associated with worse kidney function

\section{What is the key question?}

- Does fasting insulin contribute to the sex disparity in CKD and kidney function?

What are the new findings?

- Genetically predicted fasting insulin was associated with CKD and unfavourable kidney function in men but not in women

- Genetically predicted kidney function was not relevant to fasting insulin

How might this impact on clinical practice in the foreseeable future?

- Understanding the pathways underlying sex-specific effects could be valuable for drug repositioning and new drug development

\section{Introduction}

Chronic kidney disease (CKD) contributes substantially to the global burden of morbidity and mortality [1]. Kidney function declines faster in men than women, and the mortality rate is higher in men than women at all levels of pre-dialysis CKD [2], possibly due to an unhealthier, more stressful lifestyle in men than women. A protective effect of higher oestrogen in women has also been suggested [2]. A selective oestrogen receptor modulator, raloxifene, may be beneficial in albuminuria [3], with possibly an opposite role of testosterone [4], although the evidence is limited [2]. These observations suggest that the well-established evolutionary biology theory where growth and reproduction trade off against longevity, possibly in sex-specific ways [5], could be informative here, meaning factors regulating growth or reproduction could have sex-specific effects on CKD [6].

Insulin activity affects growth and reproduction [7, 8]. Insulin receptors are widespread in the kidney, and insulin activity is involved in renal tubular function [9, 10]. Observationally, fasting insulin and the homeostasis model assessment-insulin resistance index are positively associated with CKD and negatively associated with eGFR [11-13]. Observational studies are difficult to interpret because of residual confounding and potential reverse causality. Abnormal kidney function may also lead to beta cell dysfunction and insulin resistance [14]. In a recent RCT, canagliflozin, an oral sodium-glucose cotransporter 2 (SGLT2) inhibitor that lowers insulin resistance, also lowered the risk of renal failure [15], although whether the effect is wholly or partly via fasting insulin and any differences by sex remain to be clarified.
Mendelian randomisation (MR), taking advantage of genetic endowment randomly allocated at conception [16], is an increasingly popular way of testing bi-directional effects when a large RCT is unavailable or infeasible. MR examines lifetime effects of endogenous exposures, here in a generally healthy population, rather than effects of an intervention in a high-risk population [16]. As such, it provides information about risk for the majority of the population and a guide to the direction of any causal associations. Insulin resistance is closely related to hyperglycaemia; we selected between exposures based on their Bayesian posterior probability, i.e. MRBayesian model averaging (MR-BMA) [17]. To check for reverse causality, we assessed the role of kidney function in the selected exposures. Given the possibility that selective pressures are more marked in men than women $[7,8]$, we assessed the overall and sex-specific role of the selected exposure(s) and tested for differences by sex.

\section{Methods}

\section{Study design}

We used MR-BMA to identify which glycaemic trait(s) (fasting insulin, fasting glucose and/or $\mathrm{HbA}_{1 \mathrm{c}}$ ) gave the best model, overall and by sex. We used univariable or multivariable MR, as appropriate, to assess, in both directions, overall associations between the selected trait(s) and both CKD and eGFR in Chronic Kidney Disease Genetics (CKDGen) and the UK Biobank, and sex-specifically using UK Biobank individual data [18]. 


\section{The role of glycaemic traits in CKD and kidney function}

Genetic predictors for fasting insulin, fasting glucose and $\mathrm{HbA}_{1 \mathrm{c}}$ in MR-BMA We used ten SNPs (rs4846565, rs10195252, rs2943645, rs17036328, rs3822072, rs6822892, rs4865796, rs459193, rs2745353 and rs731839) for fasting insulin identified from a genome-wide association study (GWAS) conducted in 108,557 people of European ancestry (mean age 50.6 years; $~ 53 \%$ men), without diabetes [19], and tested for prediction of a comprehensive measure of insulin resistance (both euglycaemic-hyperinsulinaemic clamp- and OGTT-based measures) [20]. The genetic associations with fasting insulin (without adjustment for BMI) were obtained from the Meta-Analyses of Glucose and Insulinrelated traits Consortium (MAGIC), where mean fasting insulin averaged $\sim 56.4 \mathrm{pmol} / 1$ across studies [19]. We used 17 SNPs identified in a published GWAS of glycaemic traits from MAGIC [19] for fasting glucose (mean $\sim 5.2 \mathrm{mmol} / \mathrm{l}$ ). For $\mathrm{HbA}_{1 \mathrm{c}}$, we used 38 SNPs identified in a meta-analysis of GWAS of $\mathrm{HbA}_{1 \mathrm{c}}$ in 159,940 people from 82 cohorts, 123,665 people of European ancestry (mean age $\sim 53$ years, $\sim 48 \%$ men, mean $\mathrm{HbA}_{1 \mathrm{c}} \sim 5.4 \%$ ) [21]. The SNPs for each glycaemic trait were independent $\left(r^{2}<0.05\right.$ as seen previously [22]). To satisfy the MR assumptions, we dropped SNPs associated with potential confounders (Townsend index, smoking, alcohol drinking and physical activity) at genome-wide significance in the UK Biobank summary statistics, or in three comprehensive curated genotype to phenotype crossreference systems, Ensembl (http://www.ensembl.org/index. $\mathrm{html}$ ), the GWAS Catalog (https://www.ebi.ac.uk/gwas/) and PhenoScanner (www.phenoscanner.medschl.cam.ac.uk). The details are given in electronic supplementary material (ESM) Table 1 and ESM Table 2. For MR-BMA, we included all SNPs predicting different glycaemic traits (fasting insulin, fasting glucose or $\mathrm{HbA}_{1 \mathrm{c}}$ ), then removed duplicate SNPs and strongly correlated SNPs, using the 'clump_data' function, at a distance of $10,000 \mathrm{~kb}$ and $r^{2}$ cut-off of 0.8 , as seen previously [17]. For the remaining SNPs, we obtained their associations with fasting insulin, fasting glucose and $\mathrm{HbA}_{1 \mathrm{c}}$ from the relevant GWAS. For unavailable SNPs, we used a proxy SNP $\left(r^{2} \geq 0.8\right)$.

\section{Genetic predictors for sex-specific MR-BMA in CKD and eGFR} For sex-specific analysis of the ten SNPs predicting fasting insulin, we used those sex-specifically predicting fasting insulin in 47,806 men and 50,404 women of European ancestry without diabetes in MAGIC [23] after Bonferroni correction $(p<0.05 / 10=0.005)$, giving six SNPs each in men and women (ESM Table 1). Of the 17 SNPs predicting fasting glucose, we used those sex-specifically predicting fasting glucose in the UK Biobank (http://www.nealelab.is/blog/ 2019/9/16/biomarkers-gwas-results) and in 67,506 men and
73,089 women of European ancestry without diabetes in MAGIC, after Bonferroni correction $(p<0.05 / 17=0.003)$. Of the 38 SNPs predicting $\mathrm{HbA}_{1 \mathrm{c}}$, we used the SNPs reaching Bonferroni-corrected significance $(p<0.05 / 38=0.001)$ sex-specifically in the UK Biobank (ESM Table 1). We assessed the strength of these genetic instruments from the $F$-statistic, calculated using the square of SNP-exposure association divided by the square of its standard error [24]; SNPs with $F$-statistic $>10$ were selected.

Genetic associations with CKD and kidney function We obtained overall associations with CKD and kidney function (eGFR) from CKDGen summary statistics and overall and sex-specific associations from UK Biobank individual data (application number 42468). Sex-specific associations are not available in CKDGen.

CKDGen is a large, trans-ancestry GWAS meta-analysis comprising 60 GWAS for CKD in 625,219 people [25], 480,698 of European ancestry (41,395 CKD cases), and 121 GWAS for eGFR in 765,348 people, 567,460 of European ancestry, with median age 54 years, $50 \%$ men and median eGFR $89 \mathrm{ml} \mathrm{min}^{-1} 1.73 \mathrm{~m}^{-2}$ (IQR 81, 94) [25]. To avoid population stratification, we only included people of European ancestry. Genetic associations were obtained using logistic regression for $\mathrm{CKD}$, and linear regression for log eGFR, controlling for age, sex, genetic principal components, relatedness and other study-specific characteristics as appropriate [25].

The UK Biobank is a large, ongoing, prospective cohort study, with median follow-up of 11.1 years [18]. The UK Biobank recruited 502,713 people (aged 40-69 years, mean age 56.5 years, $45.6 \%$ men) from Great Britain from 2006 to 2010, 94\% of self-reported European ancestry. CKD events were obtained from a nurse-led interview at recruitment, and record linkage to all hospital admissions and deaths in the follow-up [18], as well as eGFR less than $60 \mathrm{ml} \mathrm{min}{ }^{-1}$ $1.73 \mathrm{~m}^{-2}$. eGFR was calculated from the Chronic Kidney Disease Epidemiology Collaboration (CKD-EPI) formula using serum creatinine [26], median $94 \mathrm{ml} \mathrm{min}{ }^{-1} 1.73 \mathrm{~m}^{-2}$ (IQR 86, 101). Genotyping was assessed using two similar arrays, i.e. the UK Biobank Lung Exome Variant Evaluation (UK BiLEVE) array and UK Biobank Axiom array. To control for population stratification, we only included participants of white British ancestry, based on self-report and genetic quality control. For quality control, we also excluded participants with (1) inconsistent self-reported and genotyped sex; (2) excess relatedness ( $>10$ putative third-degree relatives); (3) an abnormal number of sex chromosomes; or (4) poor-quality genotyping based on heterozygosity and missingness rates. After quality control, 179,917 white British men (6016 CKD cases) and 212,079 white British women (5989 CKD cases) remained. We used logistic regression to obtain SNP-specific association with CKD, controlling 
for age, sex, 20 principal components, assay array, smoking and BMI. Smoking and BMI are shared risk factors for CKD and many chronic diseases; controlling for them partly addresses selection bias from inevitably only recruiting survivors of genotype and competing risk into the underlying GWAS [27]. In contrast, we did not control for blood pressure because it may mediate associations with CKD [28, 29]. Similarly, we used linear regression to assess genetic association with log-transformed eGFR.

\section{The role of kidney function in glycaemic trait(s)}

Genetic predictors for eGFR To meet the MR assumptions, we used independent $\left(r^{2}<0.01\right)$ genetic predictors reaching genome-wide significance $\left(p<5 \times 10^{-8}\right)$ in the CKDGen GWAS meta-analysis [25]. We selected independent SNPs using the 'clump_data' function of MR-Base (http://www. mrbase.org/), and from checking the $r^{2}$ between these selected genetic variants using LD-Link (https://ldlink.nci. nih.gov/) in Europeans.

Genetic associations with glycaemic trait(s) We examined the role of eGFR in the glycaemic trait(s) identified by MR-BMA using genetic associations from the relevant MAGIC GWAS $[19,21]$, obtained using linear regression adjusted for age, sex, study site and geographic covariates in an additive genetic model [19]. For SNPs predicting eGFR not in MAGIC, proxy SNPs $\left(r^{2} \geq 0.8\right)$ were used.

\section{Statistical analysis}

Overall and sex-specific MR-BMA We used MR-BMA, a novel approach extending multivariable MR, which essentially ranks different exposure combinations on model fit (Bayesian posterior probability) [17], to select between glycaemic traits overall and sex-specifically. We standardised genetic associations with glycaemic traits from their original units to effect sizes, for ease of comparison and model selection. Standard deviations for these glycaemic traits were calculated based on MAGIC GWAS [19, 21]. As previously described [30], we used a prior probability of 0.1 and a prior variance of 0.25 . MR-BMA was also used to rank individual glycaemic traits on marginal inclusion probability (the sum of posterior probability out of all models where the glycaemic trait is present) [17]. We identified and excluded outliers, i.e. influential SNPs with very high heterogeneity (Cochran's $Q$ statistic $>100$ ) or SNPs with both large Cook's $D$ (above 0.1 ) and high heterogeneity (Cochran's $Q$ statistic $>10$ ), as previously described [30]. The analysis was repeated after removing outliers until no outlier was detected.

Overall and sex-specific associations of selected glycaemic trait(s) with CKD and eGFR Based on MR-BMA model ranking, we used the top ranked model to assess the role of the selected glycaemic trait(s). We obtained SNP-specific Wald estimates (the genetic association with CKD or eGFR divided by the genetic association with the glycaemic trait[s]) in CKDGen, and then meta-analysed these estimates using inverse variance weighting (IVW) with multiplicative random effects. If a single glycaemic trait was selected, we used genetic associations in the original units (e.g. pmol/1 for fasting insulin), rather than effect sizes, for ease of interpretation. To satisfy the MR assumptions, we checked and dropped SNP(s) associated with CKD or eGFR at genome-wide significance and detected as outliers, because the $\mathrm{SNP}(\mathrm{s})$ may be directly related to the outcome. Similarly, we conducted overall and sex-specific analyses in the UK Biobank, and meta-analysed the associations from both data sources. We used 0.05 as cutoff for significance because correction for multiple testing is more suitable for a hypothesis-generating study, such as a GWAS, than for a confirmatory study $[31,32]$. We assessed differences between sex-specific estimates (log ORs for CKD and $\beta$-coefficients for eGFR) using a $z$-test, and then obtained the two-tailed $p$ value [33].

In a sensitivity analysis, we used different methods with different assumptions, i.e. a weighted median [34] and Mendelian Randomisation Pleiotropy Residual Sum and Outlier (MR-PRESSO) with 10,000 simulations [35]. The weighted median is robust to invalid instruments and provides consistent estimation even when up to $50 \%$ of the weight is from invalid SNPs [34]. MR-PRESSO can detect and, if necessary, correct for potentially pleiotropic outliers [35], but assumes the indirect effect is independent of the direct effect. We did not use MR Egger because the limited number of SNPs means it is not very interpretable and is sensitive to outliers [36] and provides wider confidence intervals than these other methods. In a sensitivity analysis we also used Bonferroni-corrected significance $(p<0.05 / 2000$ [number of phenotypes in the UK Biobank] $=2.5 \times 10^{-5}$ ) as the cut-off for pleiotropy. If fasting insulin was the best predictor for CKD or eGFR, as hypothesised, we also checked the pattern of associations for SNP(s) in the IRS1 gene, which encodes the insulin receptor substrate 1 and is critical in insulin signalling and to the pathogenesis of type 2 diabetes [37].

Power calculations were conducted overall and by sex. The sample size needed for MR is approximately the sample size for the conventional observational study divided by the variance in the exposure explained by the SNPs [38]. Specifically, for binary outcomes, the required sample size was calculated based on the $\log \mathrm{OR}$, the ratio of cases to non-cases and the variance explained by the SNPs. For continuous outcomes, it was calculated based on the effect size and the variance explained by the SNPs.

Association of genetically predicted eGFR with selected glycaemic trait(s) We obtained SNP-specific Wald estimates 
(the association of each eGFR-related SNP with the selected glycaemic trait[s] in MR-BMA divided by the genetic association with eGFR), and meta-analysed these estimates using IVW with multiplicative random effects [39]. We used a weighted median [34] and MR-PRESSO with 10,000 simulations [35].

All statistical analyses were conducted using $\mathrm{R}$ version 3.6.2 (R Foundation for Statistical Computing, Vienna, Austria) and the R packages 'clump_data' and 'MendelianRandomization'.

\section{Ethics approval}

This research has been conducted using the UK Biobank resource under application number 42468 and publicly available data. No original data were collected for the MR study. Ethical approval for each of the studies included in the investigation can be found in the original publications (including informed consent from each participant).

\section{Results}

\section{MR-BMA model selection}

We identified ten SNPs for fasting insulin, 17 for fasting glucose and 38 for $\mathrm{HbA}_{1 \mathrm{c}}$. One SNP (rs11603334) predicted both fasting glucose and $\mathrm{HbA}_{1 \mathrm{c}}$, one SNP (rs2302593) was palindromic with allele frequency of 0.5 , two SNPs (rs9368222 and rs2383208) were excluded because of linkage equilibrium (LD) at $r^{2}$ of 0.8 and five SNPs (rs11248914, rs12621844, rs17747324, rs282587 and rs4745982) were excluded because no associations with fasting insulin were available, even for proxies (ESM Table 1). One SNP (rs1558902 predicting $\mathrm{HbA}_{1 \mathrm{c}}$ ) was associated with alcohol drinking (ESM Table 2). Given the unclear causal role of alcohol in kidney function, we kept this SNP in MR-BMA. After these exclusions, 56 SNPs remained for MR-BMA (ESM Fig. 1). No outlier was identified for CKD; three SNPs (rs4865796, rs11715915 and rs267738) were identified as outliers for eGFR and excluded. For both CKD and eGFR, fasting insulin had the highest posterior probability, and the highest marginal inclusion probability in CKDGen (Tables 1 and 2) and the UK Biobank (ESM Table 3).

For sex-specific MR-BMA, in men, we identified six SNPs predicting fasting insulin, 13 SNPs predicting fasting glucose and $37 \mathrm{SNPs}$ predicting $\mathrm{HbA}_{1 \mathrm{c}}$. In women, we identified six SNPs predicting fasting insulin, 14 SNPs predicting fasting glucose and $38 \mathrm{SNPs}$ predicting $\mathrm{HbA}_{1 \mathrm{c}}$. One SNP (rs11603334) predicted both fasting glucose and $\mathrm{HbA}_{1 \mathrm{c}}$ in men and women, one SNP (rs2302593) was palindromic with allele frequency of 0.5 , two SNPs (rs9368222 and rs2383208) were excluded because of $r^{2}>0.8$ and one SNP (rs4745982)
Table 1 The rank of models for CKD and kidney function using MRBMA in CKDGen

\begin{tabular}{cll}
\hline Rank & $\begin{array}{l}\text { Glycaemic trait or combination } \\
\text { of glycaemic traits }\end{array}$ & Posterior probability \\
\hline $\begin{array}{cll}\text { For CKD } \\
1\end{array}$ & $\beta . F I$ & 0.905 \\
2 & $\beta . F G$ & 0.039 \\
3 & $\beta . H b A_{1 c}$ & 0.029 \\
4 & $\beta . F G, \beta . F I$ & 0.015 \\
5 & $\beta . F I, \beta . H b A_{1 c}$ & 0.011 \\
For eGFR & & \\
1 & $\beta . F I$ & 0.813 \\
2 & $\beta . H b A_{1 c}$ & 0.105 \\
3 & $\beta . F G$ & 0.082 \\
\hline
\end{tabular}

Models with posterior probability $>0.01$ are listed. Three SNPs (rs4865796 with Cook's $D=0.6$ and Cochran's $Q=66.5$, rs 11715915 with Cook's $D=0.15$ and Cochran's $Q=29.1$, and rs267738 with Cochran's $Q=142.8>100$ ) were excluded for eGFR

FG, fasting glucose; FI, fasting insulin

was excluded because no sex-specific association with fasting insulin was available, even for proxies, so 51 SNPs were used for MR-BMA in men and 53 in women. After removing outliers (shown in Tables 3 and 4), fasting insulin ranked top for both men and women for both CKD and eGFR (Tables 3 and 4). All SNPs had $F$-statistics above 10 (ESM Table 1).

\section{The role of fasting insulin in CKD and kidney function}

We used univariable MR, the top ranked model in MR-BMA for the overall and sex-specific role of fasting insulin (ESM Fig. 1). Of the ten SNPs for fasting insulin, rs4865796 was

Table 2 The rank of glycaemic traits for CKD and kidney function using MR-BMA in CKDGen

\begin{tabular}{cll}
\hline Rank & Glycaemic trait & Marginal inclusion probability \\
\hline For CKD & & \\
1 & $\beta . F I$ & 0.931 \\
2 & $\beta . F G$ & 0.055 \\
3 & $\beta . \mathrm{HbA}_{1 \mathrm{c}}$ & 0.041 \\
For eGFR & & \\
1 & $\beta . \mathrm{FI}$ & 0.813 \\
2 & $\beta . \mathrm{HbA} \mathrm{A}_{1 \mathrm{c}}$ & 0.105 \\
3 & $\beta . \mathrm{FG}$ & 0.082 \\
\hline
\end{tabular}

Models with posterior probability $>0.01$ are listed. Three SNPs (rs4865796 with Cook's $D=0.6$ and Cochran's $Q=66.5$, rs 11715915 with Cook's $D=0.15$ and Cochran's $Q=29.1$, and rs267738 with Cochran's $Q=142.8>100$ ) were excluded for eGFR

$\mathrm{FG}$, fasting glucose; FI, fasting insulin 
Table 3 The rank of models for CKD and kidney function using MR-BMA in the UK Biobank by sex

\begin{tabular}{|c|c|c|c|c|c|}
\hline Rank & $\begin{array}{l}\text { Glycaemic trait or } \\
\text { combination of glycaemic traits }\end{array}$ & Posterior probability & Rank & $\begin{array}{l}\text { Glycaemic trait or } \\
\text { combination of glycaemic traits }\end{array}$ & $\begin{array}{l}\text { Posterior } \\
\text { probability }\end{array}$ \\
\hline For CKD in men & & & For CKD in women & & \\
\hline 1 & $\beta . F I$ & 0.717 & 1 & $\beta . F I$ & 0.617 \\
\hline 2 & $\beta . F G$ & 0.126 & 2 & $\beta . F G$ & 0.190 \\
\hline 3 & $\beta . \mathrm{HbA}_{1 \mathrm{c}}$ & 0.121 & 3 & $\beta . \mathrm{HbA}_{1 \mathrm{c}}$ & 0.166 \\
\hline 4 & $\beta . \mathrm{FI}, \beta . \mathrm{HbA}_{1 \mathrm{c}}$ & 0.016 & 4 & $\beta . \mathrm{FI}, \beta . \mathrm{FG}$ & 0.012 \\
\hline 5 & $\beta . F I, \beta . F G$ & 0.016 & 5 & $\beta . \mathrm{FI}, \beta . \mathrm{HbA}_{1 \mathrm{c}}$ & 0.011 \\
\hline For eGFR in men & & & For eGFR in women & & \\
\hline 1 & $\beta . F I$ & 0.585 & 1 & $\beta . F I$ & 0.487 \\
\hline 2 & $\beta . F G$ & 0.233 & 2 & $\beta . F G$ & 0.267 \\
\hline 3 & $\beta . \mathrm{HbA}_{1 \mathrm{c}}$ & 0.182 & 3 & $\beta . \mathrm{HbA}_{1 \mathrm{c}}$ & 0.245 \\
\hline
\end{tabular}

Models with posterior probability $>0.01$ are listed. One SNP (rs7756992 with Cook's $D=0.10$ and Cochran's $Q=11.4$ ) was detected as outliers in CKD in men; two SNPs (rs4865796 with Cook's $D=0.3$ and Cochran's $Q=16.5$, and rs 1558902 with Cook's $D=0.5$ and Cochran's $Q=11.2$ ) were detected as outliers in eGFR in men. No outlier was detected in CKD in women; three SNPs (rs267738 with Cook's $D=0.4$ and Cochran's $Q=93.3$, rs4865796 with Cook's $D=0.7$ and Cochran's $Q=72.8$, rs983309 with Cook's $D=0.7$ and Cochran's $Q=27.9$ ) were detected in eGFR in women

FG, fasting glucose; FI, fasting insulin

dropped for eGFR because it is strongly related to log eGFR in the UK Biobank ( $p$ value $4.7 \times 10^{-21}$; in contrast to its association with fasting insulin $p$ value $2.1 \times 10^{-8}$ ), and was detected as an outlier by MR-PRESSO. For comprehensiveness, we also excluded it in the sensitivity analysis for CKD. No SNP was related to potential confounders at genome-wide significance, but rs10195252 was related to alcohol drinking at Bonferroni-corrected significance ( $p$ value $9.4 \times 10^{-7}$ ), so it was excluded in sensitivity analysis. For sex-specific analysis, we used the six SNPs for men and six SNPs for women for CKD; after excluding rs4865796, five SNPs were used in sexspecific analysis for eGFR.

Table 4 The rank of glycaemic traits for CKD and kidney function using MR-BMA in the UK Biobank by sex
Genetically predicted fasting insulin was positively associated with CKD overall (Table 5) and in men, but not in women (Table 6). The overall association with CKD was seen for CKDGen, UK Biobank and their meta-analysis (Table 5). Genetically predicted fasting insulin was not associated with eGFR overall (Table 5), but was related to lower eGFR in men but not women (Table 6 ). The difference by sex was significant for CKD ( $p$ value 0.049 ) and eGFR ( $p$ value 0.02 ). The associations were generally robust to different analytic methods (ESM Tables 4 and 5), excluding rs4865796 for CKD (ESM Table 6) and excluding rs 10195252 (ESM Table 7). rs2943645 in the functionally relevant gene showed

\begin{tabular}{|c|c|c|c|c|c|}
\hline Rank & $\begin{array}{l}\text { Glycaemic } \\
\text { trait }\end{array}$ & $\begin{array}{l}\text { Marginal inclusion } \\
\text { probability }\end{array}$ & Rank & $\begin{array}{l}\text { Glycaemic } \\
\text { trait }\end{array}$ & $\begin{array}{l}\text { Marginal inclusion } \\
\text { probability }\end{array}$ \\
\hline $\begin{array}{l}\text { For CKD in } \\
\text { men }\end{array}$ & & & $\begin{array}{l}\text { For CKD in } \\
\text { women }\end{array}$ & & \\
\hline 1 & $\beta . F I$ & 0.750 & 1 & $\beta . F I$ & 0.640 \\
\hline 2 & $\beta . F G$ & 0.145 & 2 & $\beta . F G$ & 0.207 \\
\hline 3 & $\beta . \mathrm{HbA}_{1 \mathrm{c}}$ & 0.141 & 3 & $\beta . \mathrm{HbA}_{1 \mathrm{c}}$ & 0.181 \\
\hline $\begin{array}{l}\text { For eGFR in } \\
\text { men }\end{array}$ & & & $\begin{array}{l}\text { For eGFR in } \\
\text { women }\end{array}$ & & \\
\hline 1 & $\beta . F I$ & 0.585 & 1 & $\beta . F I$ & 0.487 \\
\hline 2 & $\beta . F G$ & 0.234 & 2 & $\beta . F G$ & 0.267 \\
\hline 3 & $\beta . \mathrm{HbA}_{1 \mathrm{c}}$ & 0.183 & 3 & $\beta . \mathrm{HbA}_{1 \mathrm{c}}$ & 0.245 \\
\hline
\end{tabular}

Models with posterior probability $>0.01$ are listed. One SNP (rs7756992 with Cook's $D=0.10$ and Cochran's $Q=11.4$ ) was detected as outliers in CKD in men; two SNPs (rs4865796 with Cook's $D=0.3$ and Cochran's $Q=$ 16.5, and rs 1558902 with Cook's $D=0.5$ and Cochran's $Q=11.2$ ) were detected as outliers in eGFR in men. No outlier was detected in CKD in women; three SNPs (rs267738 with Cook's $D=0.4$ and Cochran's $Q=93.3$, rs4865796 with Cook's $D=0.7$ and Cochran's $Q=72.8$, rs983309 with Cook's $D=0.7$ and Cochran's $Q=27.9$ ) were detected in eGFR in women

FG, fasting glucose; FI, fasting insulin 
Table 5 Overall associations of fasting insulin with CKD and kidney function using bi-directional MR

\begin{tabular}{|c|c|c|c|c|c|c|c|}
\hline Exposure & Outcome & Data sources & No. SNPs & OR & $\beta$ & $95 \% \mathrm{CI}$ & $p$ \\
\hline \multirow[t]{6}{*}{ Genetically predicted fasting insulin } & \multirow[t]{3}{*}{ CKD } & CKDGen & 10 & 2.05 & & $0.97,4.33$ & 0.06 \\
\hline & & UK Biobank & 10 & $2.51^{\mathrm{a}}$ & & $1.16,5.43$ & 0.02 \\
\hline & & Meta-analysis of CKDGen and UK Biobank & 10 & $2.23^{\mathrm{a}}$ & & $1.35,3.66$ & 0.002 \\
\hline & \multirow[t]{3}{*}{ eGFR } & CKDGen & 9 & & -0.01 & $-0.03,0.01$ & 0.31 \\
\hline & & UK Biobank & 9 & & -0.01 & $-0.03,0.02$ & 0.68 \\
\hline & & Meta-analysis of CKDGen and UK Biobank & 9 & & -0.01 & $-0.02,0.01$ & 0.29 \\
\hline Genetically predicted eGFR & Fasting insulin & MAGIC & 62 & & -0.13 & $-0.51,0.26$ & 0.52 \\
\hline
\end{tabular}

MR-PRESSO was used for genetically predicted fasting insulin and CKD in CKDGen, IVW was used for all others

a similar pattern of a positive association with $\mathrm{CKD}(\log \mathrm{OR}$ $0.07, p=0.002)$ in men but not women $(\log \mathrm{OR}-0.01, p=$ $0.53)$.

The meta-analysis of UK Biobank with CKDGen with over 53,000 cases, at an approximate $R^{2}$ of 0.01 (variance in fasting insulin explained by the genetic predictors), has 0.8 power to detect an OR of about 1.14 per standard deviation increase in the exposure [38]. The UK Biobank has 0.8 power to detect an OR of about 1.30 for CKD overall, and 1.45 sex-specifically, as well as an effect size of about 0.05 for kidney function overall, and about 0.06 sex-specifically [38].

\section{The role of kidney function in fasting insulin}

There were 264 SNPs for kidney function (eGFR) in the GWAS meta-analysis [25]. Of the 264 SNPs, 228 were uncorrelated. Of the 228 SNPs, 62 SNPs (12 SNPs and 50 proxy SNPs, ESM Table 8) were available in MAGIC. Genetically predicted eGFR was unrelated to fasting insulin (Table 3). The associations were robust to different methods (ESM Table 4).

\section{Discussion}

Our study is consistent with previous observational studies showing fasting insulin related to impaired kidney function $[10,11,13]$. It extends the evidence by confirmation using $\mathrm{MR}$, a design minimising confounding, and showing an effect specific to men. Conversely, genetically instrumented kidney function did not affect fasting insulin.

Several factors, such as kidney structure and function, lifestyle and effects of sex hormones, may explain sex differences in CKD progression [4]. For example, men and women have different renal haemodynamic responses to vasoactive agents; men are more likely to eat a high-energy diet, and to have poorer blood pressure management [4]. Sex hormones may directly affect renal function, for example, testosterone may induce glomerular podocyte apoptosis and activate the reninangiotensin system, whilst oestrogen may have opposite effects [4]. Correspondingly, fasting insulin is associated with renal haemodynamic function [40], is responsive to a highenergy diet [41] and regulates blood pressure possibly through the renin-angiotensin system [42], besides being a potential driver of growth and reproduction [7, 8]. Nevertheless, our findings are consistent with differing disease patterns by person, place and time. Our findings could be relevant to men being more susceptible to impaired kidney function [2], and possibly to Asians having a higher burden of kidney disease than people of European descent [43], because of greater insulin resistance [44]. Our findings could also be relevant to the changing disease patterns with economic development. Improvements in living conditions that enable higher levels of insulin resistance [45], with corresponding effects on kidney function, may partly explain rising rates of $\mathrm{CKD}$ that emerge with economic development, such as in China [1].

Nevertheless, our study has several limitations. First, MR is based on three assumptions (relevance, independence and
Table 6 Sex-specific associations of fasting insulin with CKD and kidney function using MR in the UK Biobank

\begin{tabular}{llllllll}
\hline Outcome & Sex & Data sources & No. SNPs & OR & $\beta$ & $95 \%$ CI & $p$ \\
\hline CKD & Men & UK Biobank & 6 & $7.23^{\text {a }}$ & & $2.46,21.2$ & $3.2 \times 10^{-4}$ \\
& Women & UK Biobank & 6 & 1.05 & & $0.21,5.21$ & 0.96 \\
\multirow{2}{*}{ eGFR } & Men & UK Biobank & 5 & & -0.04 & $-0.07,-0.01$ & 0.01 \\
& Women & UK Biobank & 5 & & 0.01 & $-0.02,0.03$ & 0.58 \\
\hline
\end{tabular}

IVW was used 
exclusion-restriction) [16]. To satisfy these assumptions, we used genetic variants from large GWAS [19, 20]. We checked for their associations with potential confounders, such as socioeconomic position and lifestyle, in the UK Biobank. Cardiovascular disease and CKD may share some risk factors, such as blood pressure and triacylglycerols, but their causal role in kidney function is unclear [46, 47]. We used twosample MR, which can be biased if samples for exposure and outcome overlap [48]. Whether some MAGIC participants also took part in the UK Biobank is unknown, but unlikely to be substantial. MAGIC and CKDGen both include participants from the TRacking Adolescents' Individual Lives Survey (TRAILS) and Ludwigshafen Risk and Cardiovascular Health (LURIC) studies, but the small overlap $(<1 \%)$ should not affect the direction of associations. CKDGen has a few participants who were patients or children (accounting for around 2.5\%), which may affect the precision, rather than the directions of association. To address the assumption of exclusion-restriction, we tested and corrected for potential pleiotropy using MR-PRESSO. We cannot exclude the possibility of a non-linear association with kidney function. A non-linear MR is ideal but not possible given fasting insulin is not available in the UK Biobank. Second, participants in the UK Biobank are healthier than the general population [49], and most do not have CKD, so our findings provide insight into the effect of a small change in exposure on population health, with corresponding public health implications. Third, our study could be affected by the inevitable selection of survivors of the exposure and of competing risk of the outcome because of the gap between randomisation at conception and subsequent recruitment. For example, if insulin resistance leads to death from myocardial infarction at 74 years [50], CKD at older ages cannot be observed, meaning the detrimental association of fasting insulin with CKD might be underestimated. We controlled for smoking and BMI, because they affect many chronic diseases and could thereby preclude recruitment into the underlying GWAS because of prior death from a competing risk, to provide better estimation. Fourth, misclassification of the outcomes is possible, but likely non-differential and so possibly biasing towards the null. Fifth, associations in Europeans may not apply to other populations, such as Asians. However, causes should be consistent although their relevance may vary by population [51]. A replication study in Asians would be worthwhile. Sixth, genetic effects might be buffered by compensatory processes or feedback mechanisms. However, such compensation would be expected to mitigate the genetic effects, thus biasing towards the null, which would not explain the positive associations of insulin resistance with CKD and kidney function.

From the perspective of clinical and public health practice, our findings suggest that fasting insulin, as well as its drivers, may be a potential target for lowering the burden of CKD.
Adiposity contributes to insulin resistance and correspondingly increases the risk of impaired kidney function [52]. Fasting insulin is also responsive to dietary factors and medications [41], such as the traditional Chinese medicine berberine [53]. Medications or diets lowering insulin resistance might be effective for the prevention and treatment of CKD, such as sodium-glucose transport inhibitors [54].

\section{Conclusions}

Genetically predicted fasting insulin was sex-specifically associated with CKD and unfavourable kidney function, whilst fasting insulin was unaffected by kidney function. Understanding the pathways underlying the sex-specific effects would be valuable, with relevance to novel treatment strategies and ensuring equally effective treatment in men and women. Replication in other studies, especially large studies in Asians, is needed.

Acknowledgements The main outcomes are from the UK Biobank under application number 42468. The authors would like to thank the UK Biobank for approving our application. Data on chronic kidney disease and kidney function have also been contributed by the CKDGen Consortium and have been downloaded from https://ckdgen.imbi.unifreiburg.de/. Genetic associations with fasting insulin, fasting glucose and $\mathrm{HbA}_{1 \mathrm{c}}$ were obtained from MAGIC, downloaded from https:// www.magicinvestigators.org/. The authors thank all investigators for sharing these data.

Data availability Access to data from the UK Biobank can be obtained by application to the UK Biobank (http://biobank.ctsu.ox.ac.uk/crystal/). The data from the CKDGen Consortium and MAGIC are publicly available, and can be downloaded from https://ckdgen.imbi.unifreiburg.de/ and https://www.magicinvestigators.org/.

Funding This research received no specific grant from any funding agency in the public, commercial or not-for-profit sectors.

Authors' relationships and activities The authors declare that there are no relationships or activities that might bias, or be perceived to bias, their work.

Contribution statement JVZ and CMS designed the study; JVZ acquired and analysed data and interpreted the results with the help of CMS; JVZ drafted the paper and CMS critically revised the paper. Both authors read and approved the final manuscript. JVZ is the guarantor of this work.

\section{References}

1. Jha V, Garcia-Garcia G, Iseki K et al (2013) Chronic kidney disease: global dimension and perspectives. Lancet 382(9888): 260-272. https://doi.org/10.1016/S0140-6736(13)60687-X

2. Carrero JJ, Hecking M, Chesnaye NC, Jager KJ (2018) Sex and gender disparities in the epidemiology and outcomes of chronic 
kidney disease. Nat Rev Nephrol 14(3):151-164. https://doi.org/10. 1038/nrneph.2017.181

3. Hadjadj S, Gourdy P, Zaoui P et al (2007) Effect of raloxifene-a selective oestrogen receptor modulator-on kidney function in post-menopausal women with type 2 diabetes: results from a randomized, placebo-controlled pilot trial. Diabet Med 24(8):906910. https://doi.org/10.1111/j.1464-5491.2007.02165.x

4. Neugarten J, Golestaneh L (2019) Influence of sex on the progression of chronic kidney disease. Mayo Clin Proc 94(7):1339-1356. https://doi.org/10.1016/j.mayocp.2018.12.024

5. Schooling CM (2016) Could androgens be relevant to partly explain why men have lower life expectancy than women? J Epidemiol Community Health 70(4):324-328. https://doi.org/10. 1136/jech-2015-206336

6. Schooling CM (2017) Practical applications of evolutionary biology in public health. Lancet 390(10109):2246. https://doi.org/10. 1016/S0140-6736(17)32462-5

7. Lin X, Yao Y, Wang B, Emlen DJ, Lavine LC (2016) Ecological trade-offs between migration and reproduction are mediated by the nutrition-sensitive insulin-signaling pathway. Int J Biol Sci 12(5): 607-616. https://doi.org/10.7150/ijbs. 14802

8. Schooling CM, Kowk MK, Zhao JV, Au Yeung SL (2018) Sulfonylureas as second line drugs in type 2 diabetes and the risk of cardiovascular and hypoglycaemic events: population based cohort study. BMJ 362:k2693

9. Singh S, Sharma R, Kumari M, Tiwari S (2019) Insulin receptors in the kidneys in health and disease. World J Nephrol 8(1):11-22. https://doi.org/10.5527/wjn.v8.i1.11

10. Artunc F, Schleicher E, Weigert C, Fritsche A, Stefan N, Haring HU (2016) The impact of insulin resistance on the kidney and vasculature. Nat Rev Nephrol 12(12):721-737. https://doi.org/10.1038/ nrneph.2016.145

11. Markus MRP, Ittermann T, Baumeister SE et al (2018) Prediabetes is associated with microalbuminuria, reduced kidney function and chronic kidney disease in the general population: the KORA (cooperative Health Research in the Augsburg Region) F4-study. Nutr Metab Cardiovasc Dis 28(3):234-242. https://doi.org/10.1016/j. numecd.2017.12.005

12. Nerpin E, Riserus U, Ingelsson E et al (2008) Insulin sensitivity measured with euglycemic clamp is independently associated with glomerular filtration rate in a community-based cohort. Diabetes Care 31(8):1550-1555. https://doi.org/10.2337/dc08-0369

13. Jang CM, Hyun YY, Lee KB, Kim H (2015) Insulin resistance is associated with the development of albuminuria in Korean subjects without diabetes. Endocrine 48(1):203-210. https://doi.org/10. 1007/s12020-014-0242-x

14. Stozer A, Hojs R, Dolensek J (2019) Beta cell functional adaptation and dysfunction in insulin resistance and the role of chronic kidney disease. Nephron 143(1):33-37. https://doi.org/10.1159/ 000495665

15. Perkovic V, Jardine MJ, Neal B et al (2019) Canagliflozin and renal outcomes in type 2 diabetes and nephropathy. N Engl J Med 380(24):2295-2306. https://doi.org/10.1056/NEJMoa1811744

16. Davies NM, Holmes MV, Davey Smith G (2018) Reading Mendelian randomisation studies: a guide, glossary, and checklist for clinicians. BMJ 362:k601

17. Zuber V, Colijn JM, Klaver C, Burgess S (2020) Selecting likely causal risk factors from high-throughput experiments using multivariable Mendelian randomization. Nat Commun 11(1):29. https:// doi.org/10.1038/s41467-019-13870-3

18. Sudlow C, Gallacher J, Allen N et al (2015) UK biobank: an open access resource for identifying the causes of a wide range of complex diseases of middle and old age. PLoS Med 12(3): e1001779. https://doi.org/10.1371/journal.pmed.1001779

19. Scott RA, Lagou V, Welch RP et al (2012) Large-scale association analyses identify new loci influencing glycemic traits and provide insight into the underlying biological pathways. Nat Genet 44(9): 991-1005. https://doi.org/10.1038/ng.2385

20. Scott RA, Fall T, Pasko D et al (2014) Common genetic variants highlight the role of insulin resistance and body fat distribution in type 2 diabetes, independent of obesity. Diabetes 63(12):4378 4387. https://doi.org/10.2337/db14-0319

21. Wheeler E, Leong A, Liu CT et al (2017) Impact of common genetic determinants of hemoglobin A1c on type 2 diabetes risk and diagnosis in ancestrally diverse populations: a transethnic genome-wide meta-analysis. PLoS Med 14(9):e1002383. https:// doi.org/10.1371/journal.pmed.1002383

22. Au Yeung SL, Luo S, Schooling CM (2018) The impact of glycated hemoglobin $\left(\mathrm{HbA}_{1 \mathrm{c}}\right)$ on cardiovascular disease risk: a Mendelian randomization study using UK Biobank. Diabetes Care 41(9): 1991-1997. https://doi.org/10.2337/dc18-0289

23. Vasiliki Lagou V, Reedik Mägi R, Hottenga JJ, et al (2019) Fasting glucose and insulin variability: sex-dimorphic genetic effects and novel loci. In Preparation. Available from https://www. magicinvestigators.org/downloads/. Accessed 8 Apr 2020

24. Bowden J, Del Greco MF, Minelli C, Davey Smith G, Sheehan NA, Thompson JR (2016) Assessing the suitability of summary data for two-sample Mendelian randomization analyses using MR-Egger regression: the role of the I2 statistic. Int J Epidemiol 45(6):19611974. https://doi.org/10.1093/ije/dyw220

25. Wuttke M, Li Y, Li M et al (2019) A catalog of genetic loci associated with kidney function from analyses of a million individuals. Nat Genet 51(6):957-972. https://doi.org/10.1038/s41588-0190407-x

26. Levey AS, Stevens LA, Schmid CH et al (2009) A new equation to estimate glomerular filtration rate. Ann Intern Med 150(9):604 612. https://doi.org/10.7326/0003-4819-150-9-200905050-00006

27. Schooling CM, Lopez P, Yang Z, Au Yeung SL, Huang JV (2020) Bias from competing risk before recruitment in Mendelian randomization studies of conditions with shared etiology. bioRxiv: https:// www.biorxiv.org/content/10.1101/716621v3.full.pdf

28. Donnelly R, Connell JM (1992) Insulin resistance: possible role in the aetiology and clinical course of hypertension. Clin Sci (Lond) 83(3):265-275. https://doi.org/10.1042/cs0830265

29. Reaven GM, Hoffman BB (1987) A role for insulin in the aetiology and course of hypertension? Lancet 2(8556):435-437. https://doi. org/10.1016/s0140-6736(87)90968-8

30. Lin BD, Alkema A, Peters T et al (2019) Assessing causal links between metabolic traits, inflammation and schizophrenia: a univariable and multivariable, bidirectional Mendelianrandomization study. Int J Epidemiol 48(5):1505-1514. https:// doi.org/10.1093/ije/dyz176

31. Perneger TV (1999) Adjusting for multiple testing in studies is less important than other concerns. BMJ 318(7193):1288. https://doi. org/10.1136/bmj.318.7193.1288a

32. Ranstam J (2019) Hypothesis-generating and confirmatory studies, Bonferroni correction, and pre-specification of trial endpoints. Acta Orthop 90(4):297. https://doi.org/10.1080/17453674.2019. 1612624

33. Paternoster R, Brame R, Mazerolle P, Piquero A (1998) Using the correct statistical test for the equality of regression coefficients. Criminology 36(4):859-866. https://doi.org/10.1111/j.1745-9125. 1998.tb01268.x

34. Bowden J, Davey Smith G, Haycock PC, Burgess S (2016) Consistent estimation in Mendelian randomization with some invalid instruments using a weighted median estimator. Genet Epidemiol 40(4):304-314. https://doi.org/10.1002/gepi.21965

35. Verbanck M, Chen CY, Neale B, Do R (2018) Detection of widespread horizontal pleiotropy in causal relationships inferred from Mendelian randomization between complex traits and diseases. Nat Genet 50(5):693-698. https://doi.org/10.1038/s41588-018-0099-7 
36. Burgess S, Thompson SG (2017) Interpreting findings from Mendelian randomization using the MR-Egger method. Eur J Epidemiol 32(5):377-389. https://doi.org/10.1007/s10654-0170255-x

37. Kovacs P, Hanson RL, Lee YH et al (2003) The role of insulin receptor substrate-1 gene (IRS1) in type 2 diabetes in Pima Indians. Diabetes 52(12):3005-3009. https://doi.org/10.2337/ diabetes.52.12.3005

38. Freeman G, Cowling BJ, Schooling CM (2013) Power and sample size calculations for Mendelian randomization studies using one genetic instrument. Int J Epidemiol 42(4):1157-1163. https://doi. org/10.1093/ije/dyt110

39. Burgess S, Butterworth A, Thompson SG (2013) Mendelian randomization analysis with multiple genetic variants using summarized data. Genet Epidemiol 37(7):658-665. https://doi. org/10.1002/gepi.21758

40. van Bommel EJM, Ruiter D, Muskiet MHA et al (2020) Insulin sensitivity and renal hemodynamic function in metformin-treated adults with type 2 diabetes and preserved renal function. Diabetes Care 43(1):228-234. https://doi.org/10.2337/dc19-1651

41. Mehran AE, Templeman NM, Brigidi GS et al (2012) Hyperinsulinemia drives diet-induced obesity independently of brain insulin production. Cell Metab 16(6):723-737. https://doi. org/10.1016/j.cmet.2012.10.019

42. Whaley-Connell A, Sowers JR (2017) Insulin resistance in kidney disease: is there a distinct role separate from that of diabetes or obesity? Cardiorenal Med 8(1):41-49. https://doi.org/10.1159/ 000479801

43. Norris KC, Agodoa LY (2005) Unraveling the racial disparities associated with kidney disease. Kidney Int 68(3):914-924. https:// doi.org/10.1111/j.1523-1755.2005.00485.x

44. Chiu KC, Cohan P, Lee NP, Chuang LM (2000) Insulin sensitivity differs among ethnic groups with a compensatory response in $\beta$-cell function. Diabetes Care 23(9):1353-1358. https://doi.org/10.2337/ diacare.23.9.1353

45. Schooling CM, Leung GM (2010) A socio-biological explanation for social disparities in non-communicable chronic diseases: the product of history? J Epidemiol Community Health 64(11):941949. https://doi.org/10.1136/jech.2008.086553
46. Lanktree MB, Theriault S, Walsh M, Pare G (2018) HDL cholesterol, LDL cholesterol, and triglycerides as risk factors for CKD: a Mendelian randomization study. Am J Kidney Dis 71(2):166-172. https://doi.org/10.1053/j.ajkd.2017.06.011

47. Yu Z, Coresh J, Qi G, et al (2019) Kidney function and blood pressure: a bi-directional Mendelian randomisation study. bioRxiv: https://www.biorxiv.org/content/10.1101/856674v1.full

48. Burgess S, Davies NM, Thompson SG (2016) Bias due to participant overlap in two-sample Mendelian randomization. Genet Epidemiol 40(7):597-608. https://doi.org/10.1002/gepi.21998

49. Fry A, Littlejohns TJ, Sudlow C et al (2017) Comparison of sociodemographic and health-related characteristics of UK Biobank participants with those of the general population. Am J Epidemiol 186(9):1026-1034. https://doi.org/10.1093/aje/kwx246

50. Kesteloot H, Decramer M (2008) Age at death from different diseases: the Flemish experience during the period 2000-2004. Acta Clin Belg 63(4):256-261. https://doi.org/10.1179/acb.2008. 047

51. Lopez PM, Subramanian SV, Schooling CM (2019) Effect measure modification conceptualized using selection diagrams as mediation by mechanisms of varying population-level relevance. J Clin Epidemiol 113:123-128. https://doi.org/10.1016/j.jclinepi.2019. 05.005

52. Chang AR, Grams ME, Ballew SH et al (2019) Adiposity and risk of decline in glomerular filtration rate: meta-analysis of individual participant data in a global consortium. BMJ 364:k5301. https://doi. org/10.1136/bmj.k5301

53. Perez-Rubio KG, Gonzalez-Ortiz M, Martinez-Abundis E, RoblesCervantes JA, Espinel-Bermudez MC (2013) Effect of berberine administration on metabolic syndrome, insulin sensitivity, and insulin secretion. Metab Syndr Relat Disord 11(5):366-369. https://doi. org/10.1089/met.2012.0183

54. Kaneto H, Obata A, Kimura T et al (2017) Beneficial effects of sodium-glucose cotransporter 2 inhibitors for preservation of pancreatic $\beta$-cell function and reduction of insulin resistance. J Diabetes 9(3):219-225. https://doi.org/10.1111/1753-0407.12494

Publisher's note Springer Nature remains neutral with regard to jurisdictional claims in published maps and institutional affiliations. 